\title{
Comprendiendo a los mejores estudiantes. Una mirada más allá de la docencia
}

\author{
Understanding the best students. A look beyond teaching
}

Entendendo os melhores estudantes. Um olhar que vai além da docência

ISSN 1688-9304 - DOI:

Gustavo Rubinsztejn Goldfarb*1

https://orcid.org/0000-0001-6656-478X

Mercedes Grijalvo Martín²

https://orcid.org/0000-0001-7885-4354

Afiliación

Pilar Rivera-Torres ${ }^{3}$

https://orcid.org/0000-0001-9319-5895

Afiliación

Luis Araújo Cazeres ${ }^{4}$

https://orcid.org/0000-0002-8713-1286

Afiliación

\section{Resumen}

Se ha demostrado que la intención de los estudiantes de recomendar su universidad está influida positivamente por su percepción de la calidad global que experimentan en el servicio universitario. Sin embargo, no está claro cuáles son las dimensiones de la calidad que más impactan en esa percepción ni cómo estas evolucionan en el tiempo para el mismo estudiante. Esta investigación pretende llenar ese vacío, utilizando medidas de calidad docente y del servicio integradas con medidas de la experiencia de los estudiantes y de su percepción de la calidad global de la carrera. El estudio utiliza modelos de ecuaciones estructurales con datos secundarios de los registros de la encuesta de calidad continua de la Universidad ORT Uruguay que siguen a 232 mejores estudiantes durante toda su experiencia con la universidad, con un total de 1681 registros. Los resultados muestran que la importancia relativa de la calidad docente y la calidad del servicio en la percepción de la calidad global evolucionan con el tiempo para los mejores estudiantes. La calidad docente es más importante en las etapas iniciales mientras que en las etapas avanzadas la calidad del servicio se vuelve más importante. Asimismo la evidencia sugiere que la etapa de la experiencia del estudiante no modera el efecto positivo de la percepción de la calidad global en la intención de recomendar. Estos resultados son de gran importancia para los responsables de las políticas universitarias. La intención de recomendar puede mejorar si la calidad docente y de los servicios está diseñada específicamente para cada etapa de la experiencia del estudiante. Esto es particularmente importante en las etapas finales, cuando los estudiantes están a punto de terminar sus estudios. Las instituciones de educación superior pueden mejorar su proceso de planificación estratégica al incluir la segmentación de los estudiantes según su experiencia.

Palabras clave: educación superior, calidad docente, calidad del servicio, intención de recomendar, buenos estudiantes, experiencia. 


\begin{abstract}
It has been documented that the students' intention to recommend their University is positively influenced by their perception on its overall quality. However, it is not clear which are the quality dimensions that impact the most on that perception, and how they evolve over time for the same student. This paper aims at filling that gap, by measuring teaching quality and quality of service, integrated with measurements of student experience and student perception of the program's overall quality. The study uses Structural Equation Models with secondary data from records of the continuous quality survey of Universidad ORT Uruguay following 232 best students over all their experience with the university, with 1681 records in total. The results show that the relative importance of teaching and service quality in the perception of overall quality evolves over time for best students. Quality of teaching is more important at initial stages of the whole student experience, while at advanced stages the quality of service becomes more important. In addition, the evidence suggests that the stage of the student experience does not moderate the positive influence of perception of overall quality on the intention to recommend their University. These results are of great importance for University policy-makers. The students' intention to recommend may be improved if teaching and services quality are designed specifically for each stage of the student experience. This is particularly important at final stages, when students are about to obtain their degrees. Higher education institutions may improve their strategic planning process by including the segmentation of students according to their experience.
\end{abstract}

Keywords: higher education, teaching quality, service quality, intention to recommend, best students, experience.

\title{
Resumo
}

Demonstrou-se que a intenção dos estudantes de indicar a universidade onde estudam é influenciada positivamente pela sua percepção quanto à qualidade global que experimentam no serviço universitário. No entanto, não fica claro quais são as dimensões da qualidade que mais impactam nessa percepção, nem como essas dimensões evoluem no tempo para um mesmo estudante. Essa pesquisa, com a intenção de preencher esse vazio, utiliza medidas de qualidade docente e de serviço, integradas a medidas da experiência dos estudantes e de sua percepção da qualidade global do curso universitário. O estudo usa modelos de equações estruturais com dados secundários dos registros da pesquisa de qualidade contínua da Universidade ORT Uruguai que acompanha 232 dentre os melhores estudantes durante toda sua experiência com a universidade, perfazendo um total de 1,681 registros. Os resultados mostram que a importância relativa da qualidade docente e da qualidade do serviço na percepção da qualidade global se acrescenta no decorrer do tempo para os melhores estudantes. A qualidade docente é mais importante nas etapas iniciais, ao passo que nas etapas mais avançadas a qualidade do serviço se torna mais relevante. Além disso, a evidência sugere que a etapa da experiência do estudante não modera o efeito positivo da percepção da qualidade global na intenção de indicar. Esses resultados são muito importantes para os responsáveis pelas políticas universitárias. A intenção de indicar pode melhorar se a qualidade docente e dos serviços estiverem projetados especificamente para cada etapa da experiência do estudante. Isto é particularmente importante nas etapas finais, quando os estudantes estão prestes a terminar seus estudos. As instituições de ensino superior podem melhorar seu processo de planejamento estratégico ao incluir a segmentação dos estudantes segundo a sua experiência.

Palavras chaves: educação superior, qualidade docente, qualidade de serviço, intenção de indicar, bons estudantes, experiência. 


\section{Introducción}

Hoy las universidades viven en un entorno muy competitivo (Sultan y Wong, 2010; Halai, 2013) y enfrentan una reducción de las ayudas a la educación pública (Quinn et al., 2009) que las obliga a ser más eficientes en su proceso de captación de nuevos estudiantes. En este contexto muchas instituciones optan por captar y retener a los mejores estudiantes (Gater, 2001) dado que ellos potencian el proceso de aprendizaje de los grupos que integran (Gibbs, 2010; Achterberg, 2005), contribuyen a obtener fondos (Helgesen y Nesset, 2007; Elsharnouby, 2016; Bradshaw, 2001) y aportan a la reputación de la universidad y a su posicionamiento en los rankings (Gater, 2001; Bradshaw, 2001). Es por ello que existe un creciente interés por comprender los factores que inciden en la elección de una universidad por parte de este tipo específico de estudiantes (Dijk, 2015; Horstschräer, 2011; Schoenherr, 2009; Chapman, 1987; Brooks, 2006; Bradshaw, 2001).

En general las investigaciones señalan que, debido a la intangibilidad del servicio educativo y su consecuente dificultad para evaluarlo, los futuros estudiantes suelen considerar la voz de terceros estudiantes en su elección universitaria (Cook y Zallocco, 1983; Mazzarol y Soutar, 2002; Marzo et al., 2005; Bruce y Edgington, 2008). Por lo tanto resulta determinante comprender cómo estos futuros estudiantes construyen su proceso de recomendación en educación superior (Mazzarol et al., 2007; Helgesen y Nesset, 2007; White, 2011). Pero aún no existe un consenso claro ni evidencia definitiva sobre los potenciales factores que pueden incidir en la intención de recomendar una universidad (Palmer et al., 2011; Chang et al., 2012).

Estudios realizados han señalado que la percepción de la calidad tiene un efecto positivo en la intención de recomendación (Teo y Soutar, 2012; Athiyaman, 1997; Paswan y Ganesha, 2009) y muchas de las investigaciones en calidad han tendido a centrarse en las dimensiones de la docencia (Ramsden, 1991; Marsh y Roche, 1993), o de los servicios facilitadores (Palmer y Holt, 2009; Marzo et al., 2005; O'Neill y Palmer, 2004), pero pocas en el abordaje multidimensional o global de la percepción de la calidad (Rubinsztejn et al., 2015; Clewes, 2003; Hill, 1995; Wang, 2012; Petruzellis et al., 2006). De los trabajos que consideran ambas dimensiones cabe destacar el de Hill (1995), quien señala que la importancia que asignan los estudiantes a la dimensión servicios facilitadores es más dinámica que la relativa a la propia docencia. En otros términos, que el peso de los determinantes de la calidad total de la educación superior varía con la experiencia que adquieren los estudiantes al avanzar en la carrera.

Sin embargo, no tenemos conocimiento de la existencia de estudios que investiguen la forma en que los buenos estudiantes universitarios perciben la calidad una vez que han ingresado a la institución y cómo esta incide en su predisposición de recomendación a futuros estudiantes. Las investigaciones señalan que en la etapa de búsqueda de la universidad los buenos estudiantes dan importancia a aspectos académicos tales como la docencia, los rankings universitarios y el hecho de que otros buenos estudiantes hayan estudiado allí (Schoenherr, 2009; Dijk, 2015), así como también servicios facilitadores tales como la financiación, el ambiente del campus y su ubicación geográfica (Vianden, 2017; Elsharnouby, 2016; Teo y Soutar, 2012; Helgesen, 2007). No obstante, no se ha analizado en profundidad la medida en que los aspectos de los servicios facilitadores siguen siendo relevantes para ellos durante su estancia en la institución, es decir, a lo largo de los años que cursan.

Retomando parcialmente algunos de los temas y resultados del estudio de Rubinsztejn et al. (2015), la presente investigación busca aportar evidencia empírica sobre las dimensiones de la calidad universitaria como antecedentes de la intención de recomendación desde 
una perspectiva multiservicio, que incluya tanto los servicios de la docencia como los servicios facilitadores, con una mirada dinámica que permita observar las percepciones de los buenos estudiantes a lo largo del proceso educativo. Con tal motivo se plantean los siguientes objetivos específicos: 1.a Identificar las dimensiones de la calidad que presentan mayor influencia en la percepción de la calidad de una carrera universitaria para los buenos estudiantes. 1.b Identificar las dimensiones de la calidad que presentan mayor influencia en la intención de recomendar una institución de educación superior para los buenos estudiantes. 2. Determinar si la influencia de dichas relaciones cambia o no en función de la experiencia acumulada por los buenos estudiantes.

A partir de la revisión de la literatura sobre servicios y de educación superior se propone un modelo que combina aspectos de ambas. En particular se adopta la mirada de Renzulli (2012) en lo que respecta a la definición de buenos estudiantes, y la de Grönroos (1990) en el abordaje de la calidad de los servicios. Este último enfoque permite integrar en el análisis la calidad de los servicios facilitadores y la calidad de la docencia como antecedentes de la calidad global de la carrera. Finalmente, se analiza la calidad global de la carrera como antecedente de la intención de recomendar una institución por parte de los buenos estudiantes introduciendo, como sugieren Clewes (2003) y Dagger y Sweeney (2007) la variable experiencia del estudiante como moderadora de dichas relaciones.

Para dar respuesta a las preguntas planteadas se presenta en primer lugar el marco conceptual en el que se encuadra esta investigación. En segundo lugar se describe la metodología empleada, detallándose la muestra, las variables y la metodología estadística. Luego se da cuenta de los principales resultados obtenidos y, por último, se presenta la discusión de los resultados así como las conclusiones y limitaciones del trabajo.

Los resultados aportan evidencia de que la percepción de la calidad global de la carrera es un antecedente positivo de la intención de recomendación de una universidad y la percepción de la calidad docente y de la calidad del servicio facilitador también presentan un efecto positivo en la percepción de la calidad global de la carrera. Respecto al dinamismo los resultados señalan una creciente importancia en el tiempo del peso de los servicios facilitadores, tanto en la percepción de la calidad global de la carrera como en la intención de recomendar la institución.

\section{Marco conceptual e hipótesis \\ La conceptualización de los buenos estudiantes}

En general los buenos estudiantes, high achieving students (Schoenherr, 2009), high ability students (Chilcutt, 2014; Dijk, 2015), honors students (Achterberg, 2005) o quality students (Gater, 2001) se definen en base a su rendimiento académico tomando en cuenta sus calificaciones. Sin embargo Renzulli (2012) distingue tres dimensiones que hacen a un buen estudiante: desempeño académico por encima del promedio, task commitment-compromiso con la tarea (que incluye aspectos como perseverancia, determinación, energía empleada y positiva) y creatividad (en términos de curiosidad, originalidad y cuestionamiento crítico). Esta mirada coincide con la perspectiva del marketing de servicios elaborada por Grönroos (1990), que entiende a los consumidores como "coproductores de los servicios" entendidos estos en sus dimensiones técnica (producto final) y funcional (proceso de producción). Cuando se aplica en el ámbito de la educación esta perspectiva permite considerar a los estudiantes como "coproductores del proceso de aprendizaje" y diferenciarlos según la calidad de su participación en términos técnicos (ej. mayor rendimiento académico, actitud cuestionadora y curiosidad) y funcionales (ej. task commitment). 
En la presente investigación se adopta esta doble mirada sobre el best student-mejor estudiante (Grönroos (1990), Renzulli (2012)) y define a los buenos estudiantes como aquellos que son buenos coproductores técnicos en el sentido de que tienen un desempeño académico superior (Grönroos, 1990; Gibbs, 2010; Brusoni et al., 2014) y que, además, son buenos coproductores funcionales en la medida en que han terminado su carrera en el tiempo preestablecido del programa o incluso menor (Chilcutt, 2014).

\section{Composición del servicio universitario}

El presente trabajo adopta la mirada de Jain et al. (2011) y delimita el alcance de la evaluación del estudiante a partir del concepto de carrera universitaria. Es decir, el conjunto de asignaturas organizadas en semestres, en un plan de estudios, y coordinadas por un equipo administrativo y de docentes al objeto de generar competencias en un área específica de conocimiento.

Desde esta perspectiva la carrera universitaria consta de diferentes tipos de actividades, que van desde las tareas en el aula hasta las actividades puramente organizativas de la enseñanza (García-Sanchis et al., 2015). Según Martínez-Argüelles et al. (2013) estas son: i) actividades del proceso central, que refieren a la docencia y se componen de actividades como clases magistrales, preparación de casos, búsqueda de artículos en la biblioteca y presentaciones orales entre otras que desarrollan profesores y alumnos en el proceso de enseñanza-aprendizaje; ii) actividades de facilitación, que refieren a los procesos organizativos y de soporte administrativo que facilitan la labor docente, tales como inscripciones a exámenes o solicitudes de documentos, cumplimiento de plazos, acceso a materiales en tiempo, reserva de laboratorios y acceso a otras infraestructuras; y iii) actividades complementarias que no son de carácter obligatorio pero añaden valor al proceso central, tales como actividades de apoyo a la inserción laboral, actividades en la cafetería y de entretenimiento, entre otras.

En este estudio se analizan la docencia (actividades del proceso central) junto con los servicios facilitadores (actividades de facilitación), y su impacto en la evaluación global de la carrera, entendida esta como la evaluación global del grado de excelencia del programa experimentado (Athiyaman, 1997). A tales efectos, se define la calidad docente como la valoración del desempeño de los docentes en base a la metodología didáctica, conocimientos transmitidos, accesibilidad y atención a los alumnos (Martínez-Argüelles et al., 2013; Gibbs, 2010). Asimismo se define la calidad del servicio facilitador como la evaluación global del grado de excelencia de los elementos administrativos y organizativos que facilitan la docencia (Grönroos, 1990; McDougall y Levesque, 1994).

Teniendo en cuenta que los buenos estudiantes necesitan que los servicios facilitadores del proceso de enseñanza-aprendizaje funcionen correctamente para satisfacer sus necesidades educativas en su carrera universitaria (Dijk, 2015; Chilcutt, 2014; Weiler, 1996), y que priorizan la existencia de un cuerpo docente de calidad en la elección de su carrera universitaria (Chapman y Jackson, 1987; Chilcutt, 2014; Spies, 1978) se plantean las siguientes hipótesis:

Hipótesis 1a) La percepción de la calidad del servicio facilitador por los buenos estudiantes es un antecedente positivo de la calidad global de la carrera.

Hipótesis 1b) La percepción de la calidad docente por los buenos estudiantes es un antecedente positivo de la calidad global de la carrera. 


\section{La recomendación y su construcción}

Athiyaman (1997) define la intención de recomendar una universidad como la predisposición que tiene un estudiante a recomendarla a partir de las experiencias e impresiones previas que ha tenido con la misma. En general, como sucede en otras industrias, los estudiantes que ya experimentaron el servicio se transforman en potenciales recomendadores, positivos o negativos, que ponen en juego la reputación de la universidad (Reiccheld, 2003) cuando intervienen en el proceso de decisión de compra de otros estudiantes pertenecientes a su círculo cercano (Kotler y Fox, 1995).

El estudio de la construcción de la intención de recomendar es particularmente relevante para las instituciones de educación superior debido a que el proceso de recomendación entre actuales y futuros estudiantes sucede principalmente a través del "boca a boca" (Stern, 1994; Swanson y Kelley, 2001). Este es uno de los medios que más impacta en la elección de una institución de educación superior (Cook y Zallocco, 1983), y puede ser incluso el factor de mayor incidencia en la generación de expectativas respecto al servicio universitario (Boyi, 2006).

Teniendo en cuenta que la percepción de la calidad tiene un efecto positivo en la intención de recomendación (Teo y Soutar, 2012; Athiyaman, 1997) y que los buenos estudiantes optan por las instituciones universitarias que ya convocaron a sus pares (Litten, 1982; Schoenherr, 2009), se plantea la siguiente hipótesis:

Hipótesis 2c: La percepción de la calidad global de la carrera por los buenos estudiantes es un antecedente positivo de la intención de recomendar la universidad.

\section{La experiencia como factor moderador}

El servicio de educación es un proceso interactivo de consumo en el cual existe una coproducción que se prolonga en el tiempo y en el que los mecanismos de creación de percepciones de calidad e intención de recomendación pueden ser fenómenos dinámicos (Brady y Cronin, 2001; Grönroos, 2001; Dagger y Sweeney, 2007).

Dentro de los avances realizados en el estudio de este componente dinámico de la evaluación de la calidad en la educación superior la investigación de Hill (1995) pone de manifiesto que los factores que influyen en los estudiantes sobre la calidad difieren de la experiencia del estudiante. En particular Hill (1995) señala que la importancia que asignan los estudiantes a la dimensión servicios facilitadores es más dinámica que la relativa a la docencia.

Sobre este antecedente, y teniendo en cuenta que la percepción de la calidad tiene un efecto positivo en la intención de recomendación (Teo y Soutar, 2012; Athiyaman, 1997), se proponen las siguientes hipótesis:

Hipótesis 3.1a) La experiencia de los buenos estudiantes modera la relación entre la percepción de la calidad del servicio facilitador y la percepción de la calidad global de la carrera.

Hipótesis 3.1b) La experiencia de los buenos estudiantes modera la relación entre la percepción de la calidad docente y la percepción de la calidad global de la carrera.

Hipótesis 3.2.c) La experiencia de los buenos estudiantes modera la relación entre la percepción de la calidad global de la carrera y la intención de recomendar la universidad. En la Figura 1 se presenta la red conceptual relativa a las hipótesis planteadas en el modelo. Se observa que tanto la percepción de la calidad docente como la percepción de la calidad del servicio facilitador pueden ser antecedentes de la percepción de la calidad global de la carrera, y que esta última puede ser antecedente de la intención de recomendar la universidad. Por tanto la variable calidad global de la carrera es una variable mediadora 
entre calidad docente y calidad del servicio facilitador respecto a la variable intención de recomendar la universidad. La moderación de la variable experiencia se concreta en su potencial efecto en todas las relaciones anteriormente postuladas.

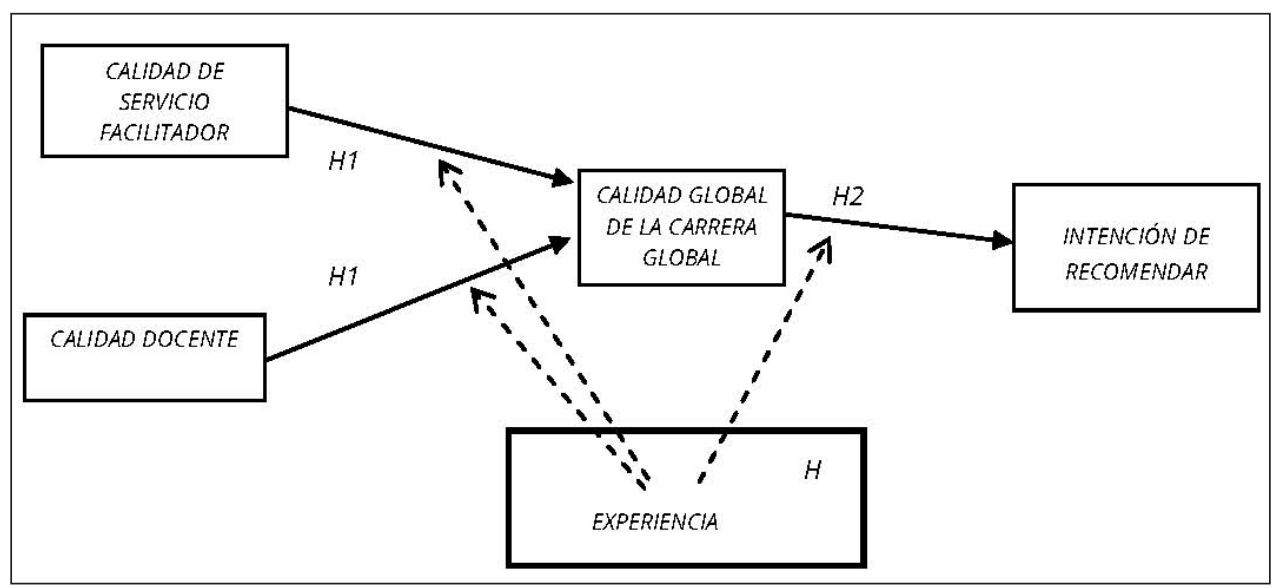

Figura 1. Red conceptual

\section{Metodología}

En esta investigación se hace uso de la Encuesta de Calidad de la Universidad ORT Uruguay, que fuera cedida a los autores para su explotación con fines de investigación, manteniendo la total confidencialidad de los estudiantes.

Se selecciona la Encuesta de Calidad de la Universidad ORT Uruguay por varias razones. La primera razón es que esta encuesta presenta, entre otras, preguntas que nos permiten operacionalizar las variables involucradas en nuestras hipótesis de trabajo. La segunda es que la base de datos secundaria resultante goza de una gran riqueza informativa. Más concretamente la base de datos original es un panel de estudiantes no balanceado que consta de un total de 195.058 observaciones o registros. Los registros de dicha base de datos están distribuidos entre un total de 7.077 estudiantes. Respecto al horizonte temporal debe señalarse que abarca 17 semestres -desde marzo de 2003 a marzo de 2011-.

Por un lado, en relación con los programas docentes, se consideran las siguientes carreras: Licenciatura en Sistemas, Licenciatura en Comunicación, Licenciatura en Economía, Ingeniería en Sistemas, Ingeniería en Electrónica/Telecomunicaciones, Licenciatura en Gerencia y Administración, Licenciatura en Diseño Gráfico, Licenciatura en Estudios Internacionales, Contador Público, Arquitectura, Licenciatura en Diseño de Interiores, Licenciatura en Diseño de Modas y Licenciatura en Diseño Industrial.

Por otro lado, respecto a las asignaturas, se consideran un total de 626 asignaturas, que se imparten en un total de 7.946 cursos, por un total de 1.195 profesores diferentes. Paralelamente se contemplan los 3 campus en los que opera esta universidad en Uruguay: Centro, Uruguay y Pocitos.

En relación con el método de recogida de la información, cabe señalar que la encuesta diseñada por la Universidad ORT Uruguay es cumplimentada obligatoriamente por sus estudiantes semestralmente (marzo y agosto), previamente a la realización de las pruebas de evaluación del semestre. Por ello, la plataforma Web de recogida de información permite identificar las valoraciones realizadas por los estudiantes a lo largo de su paso 
por la universidad (esto es, para cada observación o registro asignatura cursada se conoce el individuo i en el semestre t).

En consecuencia, esta base de datos puede ser definida como un panel no balanceado por lo que proporcionan medidas repetidas de una batería de indicadores que miden desempeño a lo largo del tiempo en el que el estudiante está cursando sus estudios universitarios. Paralelamente se dispone de información, en los diferentes semestres, sobre su intención de recomendación positiva de la institución.

Dada la información disponible en la Encuesta de Calidad de la Universidad ORT Uruguay y los objetivos de investigación descritos previamente, las variables seleccionadas para esta investigación son las siguientes: Calidad de los servicios facilitadores (C_S), Calidad docente asociada a las diferentes asignaturas que cursan cada semestre (C_D), Calidad global de la carrera que están cursando (C_G.C) e Intención de recomendación de la institución (I_R). Estas cuatro variables observadas en cada semestre se miden en una escala tipo Likert de 7 categorías (0-6), donde el 0 representa una baja puntuación y el 6 una alta puntuación. En la Tabla 1 se describen estas variables.

Tabla 1. Descripción de variables

\begin{tabular}{|c|c|c|}
\hline AUTORES & DESCRIPCIÓN & INDICADOR \\
\hline $\begin{array}{l}\text { Grönroos 1990, } \\
\text { McDougall y } \\
\text { Levesque, } 1994\end{array}$ & $\begin{array}{l}\text { Calidad de los servicios facilitadores. Evaluación de los } \\
\text { elementos administrativos y organizativos del servicio que facilitan } \\
\text { la producción y co-producción del proceso educativo. Se define } \\
\text { como la evaluación global del grado de excelencia del proceso } \\
\text { facilitador de la docencia y se mide con el indicador Califique el } \\
\text { funcionamiento de la carrera (cumplimiento de plazos, entrega } \\
\text { de materiales, organización) en el semestre t }\end{array}$ & C_S* \\
\hline $\begin{array}{l}\text { M a r tín e } z- \\
\text { Argüelles et al., } \\
2013 \\
\text { Gibbs, } 2010\end{array}$ & $\begin{array}{l}\text { Calidad docente. Evaluación global del grado de excelencia del } \\
\text { proceso de docencia, esto es, la calidad del desempeño de sus } \\
\text { docentes. Se obtiene a partir de la media de las evaluaciones } \\
\text { globales dadas a los docentes en el semestre considerado, más } \\
\text { concretamente la media del indicador Indique su evaluación global } \\
\text { del docente de la asignatura Aj en el semestre t. }\end{array}$ & C_D* \\
\hline $\begin{array}{l}\text { Athiyaman, } \\
1997\end{array}$ & $\begin{array}{l}\text { Calidad global de la carrera. Evaluación global del grado de } \\
\text { excelencia del programa que ha experimentado el estudiante y se } \\
\text { mide con el indicador Indique su evaluación global de la carrera } \\
\text { que está cursando en el semestre t. }\end{array}$ & C_G.C* \\
\hline $\begin{array}{l}\text { Athiyaman, } \\
1997\end{array}$ & $\begin{array}{l}\text { Intención de recomendación. Predisposición que tiene un } \\
\text { estudiante a recomendar un servicio a partir de las impresiones } \\
\text { previas que tiene del mismo. Se mide con el indicador Indique si } \\
\text { recomendaría la Universidad ORT para estudiar esta carrera en } \\
\text { el semestre t. }\end{array}$ & I_R* \\
\hline O'Neill, 2003 & $\begin{array}{l}\text { Experiencia del estudiante. Año en el que el estudiante } \\
\text { se encuentra en el momento de recogida de la información } \\
\text { (momento t). Se obtiene a partir de la diferencia entre la fecha de } \\
\text { encuesta y la fecha de ingreso en la Universidad, transformada } \\
\text { a años. }\end{array}$ & $E X P_{-} * *$ \\
\hline
\end{tabular}

* Las escalas utilizadas son de tipo Likert de 7 categorías (0-6), en donde el 0 representa una baja puntuación y el 6 alta.

** Variable con 6 categorías, de 1 a 6 (EXP_1, EXP_2, EXP_3, EXP_4,EXP_5 y EXP_6), donde 
los primeros cinco valores representan los años que lleva el estudiante en la institución y el 6 da cuenta de aquellos que superan dicho valor (más de 5 años).

Asimismo cabe señalar que de acuerdo al sistema de recolección de información diseñado por la Universidad ORT Uruguay los estudiantes disponen de códigos estables de entrada en el sistema. Por ello en la base de datos se encuentran tanto variables dinámicas como estáticas relacionadas con las características propias del estudiante. Entre las variables estáticas destacan: la fecha de nacimiento, la fecha de ingreso en la universidad, la carrera cursada, el campus, el sexo y si procedía la fecha de graduación del estudiante. Mientras que entre las variables dinámicas destaca, por su relevancia para esta investigación, la fecha de realización de la encuesta, es decir, el semestre al que corresponde cada uno de los registros considerados.

Por supuesto existía información referente a los códigos de asignatura, curso y docente así como sobre el rendimiento del estudiante en cada uno de los registros en los que estaba implicado y las asignaturas que ha cursado. Esta última variable, rendimiento de estudiante, aunque muy atractiva finalmente no fue seleccionada por su alta tasa de datos ausentes (70\%). Esta alta tasa se debió al sistema de evaluación propio de esta institución universitaria, donde la convalidación de asignaturas implica la no existencia de nota explícita.

\section{Depuración de la base de datos y construcción de variables}

Una vez seleccionadas las variables relevantes para esta investigación dentro de la base de datos original, se procede a depurar dicha base de datos mediante las variables estáticas del estudiante, esto es, aquellas que en un principio no pueden variar a lo largo de los semestres en los que se cumplimentan las encuestas de evaluación.

En primer lugar se procede a analizar los valores anómalos tanto en términos de código de estudiante como de sus registros. Entre otros controles se analiza la unicidad entre código de estudiante y valor en las variables sexo y fecha de nacimiento. Paralelamente se controla dicha unicidad con las variables fecha de ingreso, carrera cursada, campus, graduación y fecha de graduación (si procede).

Así, entre los filtros aplicados cabe señalar que se restringió la fecha de ingreso del estudiante a una única fecha, y se eliminaron aquellos estudiantes que presentaban diferentes fechas de ingreso en la universidad. Tras dichos controles se calculó la edad de entrada del estudiante en la Universidad ORT de Uruguay. Se procedió de igual forma con las variables carrera cursada, campus, graduación y fecha de graduación (si procede). Esto es, se aplicó la decisión de que la eliminación de un estudiante implicaba la eliminación de todos los registros asociados al mismo código de estudiante.

En segundo lugar, respecto a las variables dinámicas, cabe destacar que se eliminaron todos los registros que no estuvieran asociados a una asignatura, curso y/o docente. En este caso no se procedió a la eliminación del estudiante sino simplemente a la eliminación de los registros que presentaban valor ausente en dichas variables.

En tercer lugar, dada la estructura anidada de los datos se agregaron los registros en términos de semestres. Para cada semestre se dispone de las percepciones de la calidad de los servicios facilitadores prestados por la universidad (C_S), de la calidad global de la carrera (C_G.C), de su intención de recomendar a la universidad (I_R) y de una valoración media de la calidad de la docencia recibida en cada uno de los semestres considerados 
(C_D). Esta última se calcula a partir de las valoraciones dadas por el estudiante a los distintos profesores que impartieron clase en cada uno de dichos semestres.

Tras anidar los registros en semestres se procedió a depurar los casos ausentes mediante el método listwise deletion. Por lo que, tras depurar la base de datos, la muestra objeto de análisis presenta 30.590 registros obtenidos a partir de 6.409 estudiantes.

A partir de la aplicación de los controles señalados y la correspondiente depuración de estudiantes y registros se construye una nueva variable dinámica que recoge la experiencia del estudiante en su paso por la universidad. Dado que se disponía de la fecha de realización de la encuesta (para cada uno de los registros) y de la fecha de entrada del estudiante en la universidad (fecha única), la simple sustracción entre ambas variables nos proporciona una variable proxy de la experiencia que va teniendo el estudiante en cada semestre con la universidad en la que está cursando sus estudios. En la Tabla 2 se describe la variable experiencia del estudiante con la universidad. Por lo tanto, la variable experiencia del estudiante (EXP_) se construye restando de la fecha de encuesta la fecha de ingreso del mismo en la universidad y posteriormente se transforma en años de experiencia. De esta forma se obtiene una variable con 4 categorías de respuesta, de 1 a 4 , que consignamos como EXP_1, EXP_2, EXP_3 y EXP_4.

Respecto a la identificación de los buenos estudiantes de la Universidad ORT de Uruguay, tal como se ha indicado se disponía de información sobre el rendimiento del estudiante en cada una de las asignaturas que había cursado. Lamentablemente esta variable no pudo ser utilizada por la alta presencia de datos ausentes (sistema de convalidación de asignaturas). Por ello se optó por considerar como buenos estudiantes a aquellos alumnos que cumplían dos condiciones: haber obtenido su graduación en el período considerado (699 estudiantes y 5.658 registros) y haber finalizado sus estudios en 4 años o menos (232 estudiantes y 1.681 registros) (ver Tabla 2$)$.

Tabla 2. Tipología de estudiantes y experiencia en la universidad.

\begin{tabular}{|c|c|c|c|c|c|c|c|c|}
\hline $\begin{array}{c}\text { Experiencia } \\
\text { del } \\
\text { estudiante }\end{array}$ & \multicolumn{2}{|c|}{$\begin{array}{c}\text { Total } \\
\text { estudiantes }\end{array}$} & \multicolumn{2}{c|}{$\begin{array}{c}\text { Estudiantes } \\
\text { no graduados }\end{array}$} & \multicolumn{2}{c|}{$\begin{array}{c}\text { Estudiantes } \\
\text { graduados }\end{array}$} & \multicolumn{2}{c|}{$\begin{array}{c}\text { Buenos } \\
\text { estudiantes }\end{array}$} \\
\hline Registros & Estudiantes & Registros & Estudiantes & Registros & Estudiantes & Registros & Estudiantes \\
\hline 1 año (EXP_1) & 9957 & 6199 & 8719 & 5509 & 1238 & 690 & 428 & 231 \\
\hline $\begin{array}{c}2 \text { años } \\
\text { (EXP_2) }\end{array}$ & 7578 & 4516 & 6314 & 3843 & 1264 & 673 & 413 & 221 \\
\hline $\begin{array}{c}\text { 3 años } \\
\text { (EXP_3) }\end{array}$ & 5522 & 3384 & 4322 & 2722 & 1200 & 662 & 402 & 224 \\
\hline $\begin{array}{c}4 \text { años } \\
\text { (EXP_4) }\end{array}$ & 3925 & 2477 & 2810 & 1855 & 1115 & 622 & 438 & 209 \\
\hline $\begin{array}{c}5 \text { años } \\
\text { (EXP_5) }\end{array}$ & 2156 & 1497 & 1542 & 1082 & 614 & 415 & - & - \\
\hline $\begin{array}{c}\text { Más de 5 } \\
\text { años (EXP_6) }\end{array}$ & 1452 & 750 & 1225 & 610 & 227 & 140 & - & - \\
\hline Total & $\mathbf{3 0 5 9 0}$ & $\mathbf{6 4 0 9}$ & $\mathbf{2 4 9 3 2}$ & $\mathbf{5 7 1 0}$ & $\mathbf{5 6 5 8}$ & $\mathbf{6 9 9}$ & $\mathbf{1 6 8 1}$ & $\mathbf{2 3 2}$ \\
\hline
\end{tabular}

En la Tabla 3 se presentan los perfiles descriptivos de la muestra total respecto de la muestra de los buenos estudiantes en la Universidad ORT. Esto es, se da cuenta del perfil de este tipo de estudiantes respecto al perfil general. Destaca la asociación positiva entre el porcentaje de mujeres y de buenos estudiantes (42.2\% frente a $65.9 \% ; \chi 2[1]=9.8 p<.00$ ). 
Respecto a macro área existen diferencias entre ambos perfiles ( $\chi 2[3]=33.2 p<.00)$; relación positiva con Sociales (36.6\% frente a 59.5\%) y Diseño (17.1\% frente a 30.2\%) y relación negativa con Ingeniería (28.7\% frente a $6.9 \%$ ) y otros programas (17.6\% frente a $3.4 \%$ ).

Tabla 3. Descripción de la muestra

\begin{tabular}{|c|c|c|c|c|c|c|c|c|}
\hline & \multicolumn{4}{|c|}{ Estudiantes } & \multicolumn{4}{|c|}{ Buenos estudiantes } \\
\hline & Registros & & Estudiantes & & Registros & & Estudiantes & \\
\hline & $\mathbf{N}$ & $\%$ & $\mathbf{N}$ & $\%$ & $\mathbf{N}$ & $\%$ & $\mathbf{N}$ & $\%$ \\
\hline \multicolumn{9}{|l|}{ Sexo } \\
\hline Masculino & 16881 & 55,2 & 3578 & 55,8 & 565 & 33.6 & 79 & 34.1 \\
\hline Femenino & 13709 & 44,8 & 2831 & 44,2 & 1116 & 66.4 & 153 & 65.9 \\
\hline \multicolumn{9}{|c|}{ Edad de ingreso } \\
\hline 17 años & 2413 & 7,9 & 440 & 6,9 & 182 & 10.8 & 24 & 10.3 \\
\hline 18 años & 8689 & 28,4 & 1770 & 27,6 & 567 & 33.7 & 80 & 34.5 \\
\hline 19 años & 5728 & 18,7 & 1268 & 19,8 & 393 & 23.4 & 55 & 23.7 \\
\hline $\begin{array}{c}\text { De } 20 \text { a } 22 \\
\text { años }\end{array}$ & 7364 & 24,1 & 1474 & 23,0 & 318 & 18.9 & 43 & 18.5 \\
\hline $\begin{array}{c}\text { De } 23 \text { a } 25 \\
\text { años }\end{array}$ & 3182 & 10,4 & 702 & 11,0 & 94 & 5.6 & 14 & 6.0 \\
\hline $\begin{array}{c}\text { De } 26 \text { a } 30 \\
\text { años }\end{array}$ & 2103 & 6,9 & 500 & 7,8 & 57 & 3.4 & 7 & 3.0 \\
\hline $\begin{array}{c}\text { Más de } 30 \\
\text { años }\end{array}$ & 1111 & 3,6 & 255 & 4,0 & 70 & 4.2 & 9 & 3.9 \\
\hline \multicolumn{9}{|l|}{ Macro-área } \\
\hline Ingeniería & 8854 & 28,9 & 1838 & 28,7 & 108 & 6.4 & 16 & 6.9 \\
\hline Sociales & 11115 & 36,3 & 2344 & 36,6 & 1026 & 61.0 & 138 & 59.5 \\
\hline Diseño & 5163 & 16,9 & 1096 & 17,1 & 481 & 28.6 & 70 & 30.2 \\
\hline Otras & 5458 & 17,8 & 1131 & 17,6 & 66 & 3.9 & 8 & 3.4 \\
\hline
\end{tabular}

\section{Análisis de la información}

Al objeto de alcanzar los objetivos marcados en esta investigación, en primer lugar se realiza un análisis descriptivo de las variables clave, en términos de sus medidas de posición y su matriz de correlaciones. Dicho análisis se complementa con un Análisis de la Varianza (ANOVA).

Respecto al contraste de las hipótesis planteadas señalar que la metodología utilizada se concreta en los Modelos de Ecuaciones Estructurales. Esta aproximación metodológica proporciona una serie de ventajas con respecto a otras aproximaciones. Una de las más importantes es que permite al investigador introducir información a priori y valorar su inclusión, además de reformular las modelizaciones propuestas desde una perspectiva multi-muestra (Bollen, 1989; Jöreskog, 1993).

En esta investigación se estiman los modelos con MPLUS 7 (Muthén y Muthén, 1998-2012) y EQS 6.2 (Bentler, 2008). Más concretamente, los modelos se estiman mediante Máxima Verosimilitud Robusta, a la no normalidad y la existencia de grupos (estudiantes), así se establecen las correcciones propuestas por Satorra (1992) tanto en las estimaciones de los errores estándares de los parámetros estimados como en los estadísticos de bondad de ajuste (Asparouhov, 2005; Muthén y Muthén, 1998-2012).

A efectos de valorar el ajuste de los modelos, se presentan diferentes estadísticos e índices de bondad de ajuste. Más concretamente, se da cuenta del estadístico Robust-x2, el Root 
Mean Square Error of Approximation (RMSEA), el Standardised Root Mean Square Residual (SRMR) y el Comparative Fit Index (CFI) (Bollen, 1998; Browne et al., 1993; Hu y Bentler, 1999; Jöreskog y Sörbom, 1996). Un RMSEA en el rango 0,05-0,10 es indicativo de un ajuste razonable (Hu y Bentler, 1999). El índice SRMR está en el intervalo -0 y 1-, valores del índice inferiores a 0,05 indican un buen ajuste del modelo, mientras que valores inferiores a 0,08 son indicativos de la existencia de un ajuste razonable (Bollen, 1989; Hair et al., 2006). Valores del índice CFI mayores a 0,90 son indicativos de un ajuste razonable (Hooper et al., 2008).

Con el objetivo de testar el modelo planteado (Figura 1), se operacionaliza un modelo de ecuaciones simultáneas. Este modelo recoge la moderación de la variable experiencia del estudiante como variable categórica, lo que implica la necesidad de plantear un análisis multi-muestra. Más concretamente, para el contraste de las hipótesis planteadas, en primer lugar se procede a estimar el modelo de ecuaciones simultáneas, poniendo a prueba el modelo estructural sin considerar la variable experiencia. Así, se procede a valorar la significatividad individual de los parámetros estructurales, así como la significatividad de los efectos directos e indirectos.

El modelo resultante, tras contrastar la significatividad individual de los parámetros estructurales, será el modelo de partida del análisis multi-muestra. Por lo que una vez testeado dicho modelo inicial, a efectos de valorar la moderación de la variable experiencia, se procede de la misma forma en las submuestras definidas por dicha variable.

Respecto al contraste de la Hipótesis 3 "valorar la moderación de la variable experiencia" se estima dicho modelo en las cuatro submuestras (EXP_1, EXP_2, EXP_3 y EXP_4).

\section{Descripción de variables y contraste de hipótesis}

En primer lugar en la Tabla 4 se presentan los estadísticos descriptivos, la matriz de correlaciones entre las variables de interés y los resultados de los contrastes de igualdad de medias atendiendo a la variable experiencia del estudiante en la muestra total (1.681 registros anidados en 232 estudiantes). Seguidamente, en la Tabla 5 se da cuenta de los parámetros estimados (Figura 1) y en la Tabla 6 de los resultados de los contrastes de igualdad de parámetros en torno a la variable experiencia del estudiante.

En la Tabla 4 se presentan los estadísticos descriptivos de las variables. En primer lugar cabe señalar que las medias superan en todas las variables el 4,00, en una escala tipo Likert de 7 categorías (0-6), por lo que los estudiantes perciben altos niveles de calidad del servicio facilitador (C_S: 4,53), de la docencia (C_D: 4,65) y de la carrera (C_G.C: 4,61). Paralelamente se observan altos niveles de intención de recomendación de la Universidad (I_R: 4,88). Como en la Muestra Total cabe señalar que la matriz de correlaciones entre las variables pone de manifiesto que las correlaciones entre las variables C_S, C_D, C_G.C e I_R son todas positivas y significativas ( $p$-valor $<0,01$ ), mientras que se observan correlaciones negativas entre dichas variables y la variable experiencia del estudiante ( $p$-valor $<0,10)$. En este caso la variable experiencia consta únicamente de cuatro categorías (EXP_1, EXP_2, EXP_3 y EXP_4).

A efectos de analizar con mayor detalle estas relaciones negativas (experiencia del buen estudiante respecto a las variables de interés) se realiza un análisis de la varianza (ANOVA), calculando y testeando la igualdad de medias de C_S, C_D, C_G.C e I_R en las categorías de la variable experiencia (EXP_1, EXP_2, EXP_3 y EXP_4). Los resultados de este análisis ponen de manifiesto que sí existe cierta tendencia negativa pero que no es lineal ni igual en todas las variables objetivo. Los niveles de calidad y de intención de recomendación de la universidad descienden levemente conforme aumenta la experiencia del estudiante con la Universidad. 
Al objeto de testear las Hipótesis 1a, 1 b y 2c se valora un modelo de ecuaciones simultáneas no saturado (Figura 1), con 2 grados de libertad en la muestra definida por los buenos estudiantes. En la Tabla 5 se presentan los estadísticos de ajuste global del modelo, los que muestran un ajuste razonable del modelo estimado $(\chi 2[2]=27.24, \mathrm{RMSEA}=.07, \mathrm{SRMR}=$ $.03, \mathrm{CFI}=.98)$.

Tabla 4. Estadísticos descriptivos, matriz de correlaciones y relaciones con experiencia

\begin{tabular}{|c|c|c|c|c|c|c|c|c|c|c|}
\hline & \multirow[t]{2}{*}{ MEDIA } & \multicolumn{4}{|c|}{ MATRIZ DE CORRELACIONES } & \multicolumn{4}{|c|}{ VALORACIONES MEDIAS/EXPERIENCIA } & \multirow[t]{2}{*}{ ANOVA } \\
\hline & & C_S & C_D & C_G. & C I_R & EXP_1 & EXP_2 & EXP_3 & EXP_4 & \\
\hline $\begin{array}{l}\text { Calidad de } \\
\text { servicio } \\
\text { (C_S) }\end{array}$ & 4,53 & & & & & $4,74 \downarrow$ & 4,49 & $4,35 \downarrow$ & 4,54 & $10,02 * * *$ \\
\hline $\begin{array}{l}\text { Calidad } \\
\text { docente } \\
\text { (C_D) }\end{array}$ & 4,65 & $0,38 * * *$ & & & & 4,70 & 4,61 & 4,60 & 4,69 & $2,17^{*}$ \\
\hline $\begin{array}{l}\text { Calidad } \\
\text { global de } \\
\text { la carrera } \\
\text { (C_G.C) }\end{array}$ & 4,61 & $0,57 * \star \star$ & $0,48^{* * *}$ & & & $4,71 \downarrow$ & 4,62 & $4,55 \downarrow$ & 4,56 & $2,86^{\star *}$ \\
\hline $\begin{array}{l}\text { Intención de } \\
\text { recomendar } \\
\text { (I_R) }\end{array}$ & 4,88 & $0,40 * * *$ & $0,34 * * *$ & $0,55^{* \star *}$ & & $5,00 \downarrow$ & 4,88 & $4,79 \downarrow$ & 4,83 & $3,10^{* *}$ \\
\hline $\begin{array}{l}\text { Experiencia } \\
\text { del } \\
\text { estudiante } \\
\text { (EXP) }\end{array}$ & & $-0,07 * \star \star$ & 0,00 & $-0,06 * *$ &,$- 06 * *$ & & & & & \\
\hline
\end{tabular}

*** p-valor<0,01; **p-valor<0,05; *p-valor<0,10.

En la Tabla 5 se sintetizan los principales resultados de los modelos de ecuaciones simultáneas estimados como en el caso anterior, tanto para todos los buenos estudiantes como para las 4 submuestras definidas por la variable experiencia. Respecto a los buenos estudiantes en general cabe señalar que los porcentajes de varianza explicada tanto de la variable calidad global de la carrera $\left(R^{2}=0,40\right)$ como de la variable intención de recomendación son significativos $\left(R^{2}=0,31\right)$. Paralelamente puede destacarse que no existe evidencia para el rechazo de las tres primeras hipótesis planteadas (Hipótesis 1a, Hipótesis 1 b e Hipótesis 2c). Tanto la calidad de servicio (Hipótesis 1a: 0,37, p-valor<0.00) como la calidad docente (Hipótesis 1b: 0,37, p-valor $<0.00$ ) son antecedentes positivos de las percepciones de calidad global de la carrera de los estudiantes. Paralelamente dichos componentes de la calidad (servicios facilitadores y docencia) son antecedentes indirectos $(0,26$ y 0,26, p-valor $<0,00)$ de la intención de recomendación positiva de la universidad. Respecto al papel que desempeña la calidad global de la carrera se puede señalar que también es un antecedente positivo de la intención de recomendación (Hipótesis 2c: 0,69 , p-valor $<0.00$ ) y juega un papel mediador de los componentes de la calidad (servicio facilitador y docencia) respecto de la intención de recomendación positiva de la universidad. Por lo tanto los resultados obtenidos nos permiten no rechazar las hipótesis $1 \mathrm{a}, 1 \mathrm{~b}$ y $2 \mathrm{c}$. En este caso, como en el caso anterior, del análisis de los efectos directos e indirectos (Tabla 5) se desprende que las tres variables consideradas son clave para gestionar la imagen de la universidad, medida a partir de la intención de recomendación del estudiante. La variable clave se concreta en las percepciones de calidad global de la carrera que el estudiante está cursando $(0,69, \mathrm{p}$-valor $<0.00)$. Asimismo se aprecia que es tan importante el servicio principal que ofrece la universidad -docencia- $(0,26, \mathrm{p}$-valor $<0.00)$ como la calidad de los servicios facilitadores $(0,26, \mathrm{p}$-valor<0.00). 
Respecto al papel moderador de la variable experiencia -Hipótesis 3-, como en el caso general, también se detectaron leves diferencias aunque significativas en términos de las medidas de posición de las variables de interés. Al analizar los resultados de los modelos de ecuaciones simultáneas en las cuatro submuestras definidas por la variable experiencia del estudiante, en primer lugar se observa que los estadísticos de bondad de ajuste del modelo nos permiten no rechazarlos. Dichos estadísticos e índices evidencian un ajuste razonable para cada una de las submuestras $\left(\mathrm{EXP}_{-} 1: \chi 2[2]=7.42, \mathrm{RMSEA}=.08, \mathrm{SRMR}=\right.$ $.03, \mathrm{CFI}=.97 ; \mathrm{EXP} 2: \chi 2[2]=24.18, \mathrm{RMSEA}=.08, \mathrm{SRMR}=.05, \mathrm{CFI}=.94 ; \mathrm{EXP} \_3: \chi 2[2]=11.42$, RMSEA $=.08, \mathrm{SRMR}=.03, \mathrm{CFI}=.97$; EXP_4: $\chi 2[2]=1.87, \mathrm{RMSEA}=.00, \mathrm{SRMR}=.02, \mathrm{CFI}=.99$ ).

No rechazados los modelos estimados en las cuatro submuestras se analizan a continuación los parámetros estimados. En primer lugar, se observa que los porcentajes de varianza explicada tanto de la variable calidad global de la carrera $\left(R^{2}=0,31 / 0,38 / 0,47 / 0,48\right)$ como de la variable intención de recomendación son significativos $\left(R^{2}=0,28 / 0,32 / 0,29 / 0,32\right)$. En segundo lugar, que los diferentes parámetros estimados varían conforme aumenta la experiencia del estudiante (Tabla 5). Estas diferencias muestrales son juzgadas mediante contrastes de igualdad de parámetros atendiendo a la experiencia del estudiante.

Tabla 5. Parámetros estimados

\begin{tabular}{|c|c|c|c|c|c|}
\hline TOTAL: 1681 (232) & $C_{-} S$ & $C_{-} D$ & C_G.C & R2 & \\
\hline \multirow[t]{2}{*}{ Efectos directos } & C_G.C & $0,37 * \star *$ & $0,37^{\star \star \star}$ & & 0,40 \\
\hline & I_R & - & - & $0,69 * * *$ & 0,31 \\
\hline Efectos indirectos & I_R & $0,26 * * *$ & 0,26 *** & - & \\
\hline EXP_1: $428(231)$ & C_S & $C_{-} D$ & C_G.C & $\mathrm{R} 2$ & \\
\hline \multirow[t]{2}{*}{ Efectos directos } & C_G.C & $0,26 * \star \star$ & $0,40 * \star \star$ & & 0,31 \\
\hline & I_R & - & - & $0,64 * * *$ & 0,28 \\
\hline Efectos indirectos & I_R & $0,17 * \star *$ & $0,26 * * *$ & - & \\
\hline EXP_2: $413(221)$ & & C_S & C_D & C_G.C & R2 \\
\hline \multirow[t]{2}{*}{ Efectos directos } & C_G.C & $0,29 * \star *$ & $0,44 * * *$ & & 0,38 \\
\hline & I_R & - & - & $0,71 * * \star$ & 0,32 \\
\hline Efectos indirectos & I_R & $0,21 * * *$ & $0,31 * * *$ & - & \\
\hline EXP_3: 402 (224) & C_S & C_D & C_G.C & R2 & \\
\hline \multirow[t]{2}{*}{ Efectos directos } & C_G.C & $0,40 * * *$ & $0,40 * * *$ & & 0,47 \\
\hline & I_R & - & - & $0,72 * \star \star$ & 0,29 \\
\hline Efectos indirectos & I_R & $0,29 * \star *$ & $0,28 * * *$ & - & \\
\hline EXP_4: 438 (209) & C_S & C_D & C_G.C & R2 & \\
\hline \multirow[t]{2}{*}{ Efectos directos } & C_G.C & $0,53^{* * *}$ & $0,30 * * *$ & & 0,48 \\
\hline & I_R & - & - & $0,68 * * \star$ & 0,32 \\
\hline Efectos indirectos & I_R & $0,36^{* * *}$ & $0,20 * * *$ & - & \\
\hline
\end{tabular}

*** p-valor $<0,01 ; * *$ p-valor $<0,05 ; *$ p-valor $<0,10$.

TOTAL: $\chi 2[2]=27.24, \mathrm{RMSEA}=0.07, \mathrm{SRMR}=0.03, \mathrm{CFI}=0.98$

EXP_1: $\chi 2[2]=7.42$, RMSEA $=0.08$, SRMR $=0.03, \mathrm{CFI}=0.97$

EXP_2: $X 2[2]=24.18, \mathrm{RMSEA}=0.08, \mathrm{SRMR}=0.05, \mathrm{CFI}=0.94$

EXP_3: $X 2[2]=11.42, \mathrm{RMSEA}=0.08, \mathrm{SRMR}=0.03, \mathrm{CFI}=0.97$

EXP_4: $\chi 2[2]=1.87$, RMSEA $=0.00$, SRMR $=0.02, C F I=0.99$ 
En la Tabla 6 se sintetizan los principales resultados de dichos contrastes dos a dos.

Tabla 6. Contrastes de igualdad de parámetros.

\begin{tabular}{|c|c|c|c|c|c|c|c|}
\hline & \multicolumn{2}{|c|}{ EXP_1 } & \multicolumn{2}{|c|}{ EXP_2 } & \multicolumn{2}{|c|}{ EXP_3 } & EXP_4 \\
\hline EXP.1 & & & $x^{2}[7]=29.44$ & & $\begin{array}{l}\chi^{2}[7]=24.49 \\
\chi^{2}[6]=18.01\end{array}$ & & $\begin{array}{l}X^{2}[7]=26.64 \\
X^{2}[6]=9.88\end{array}$ \\
\hline EXP_2 & $\chi^{2}[1]$ & $p$-valor & & & $\begin{array}{l}\chi^{2}[7]=34.21 \\
\chi^{2}[6]=32.12\end{array}$ & & $\begin{array}{l}\chi^{2}[7]=34.47 \\
\chi^{2}[6]=23.22 \\
\chi^{2}[5]=20.02\end{array}$ \\
\hline C_S $\rightarrow \quad$ C_G.C & 0,67 & & & & & & \\
\hline$C_{-} D \rightarrow C_{-} G . C$ & 0,49 & & & & & & \\
\hline C_G.C $\rightarrow$ I_R & 0,60 & & & & & & \\
\hline EXP_3 & $\chi^{2}[1]$ & p-valor & $\chi^{2}[1]$ & p-valor & & & $X^{2}[7]=14.82$ \\
\hline$C_{-} S \rightarrow C_{-}$G.C & 5,33 &.$* *$ & 2,99 & * & & & \\
\hline$C_{-} D \rightarrow C_{-} G . C$ & 0,01 & & 0,32 & & & & \\
\hline C_G.C $\rightarrow$ I_R & 0,65 & & 0,01 & & & & \\
\hline EXP_4 & $\chi^{2}[1]$ & p-valor & $\chi^{2}[1]$ & $p$-valor & $\chi^{2}[1]$ & p-valor & \\
\hline$C_{-} S \rightarrow C_{-}$G.C & 13,67 & $* \star *$ & 10,15 & $* * *$ & 2,29 & & \\
\hline$C_{-} D \rightarrow C_{-} G . C$ & 1,35 & & 3,05 & * & 0,16 & & \\
\hline C_G.C $\rightarrow$ I_R & 0,14 & & 0,14 & & 0,19 & & \\
\hline
\end{tabular}

*** p-valor<0,01; **p-valor<0,05; *p-valor<0,10.

En la parte inferior de la diagonal se encuentran los estadísticos $\chi 2[1]$ correspondientes al contraste de igualdad del parámetro fila.

En la parte superior de la diagonal se encuentran los estadísticos $\chi 2[i]$ correspondientes al contraste de igualdad del vector de parámetros de dimensión i.

Dados los resultados obtenidos tras realizar los contrastes de igualdad de parámetros (Tabla 6) cabe señalar que la variable experiencia modera algunas de las relaciones existentes entre la calidad del servicio y la percepción del estudiante sobre la calidad global de la carrera que cursa (Hipótesis 3.1a). Más concretamente, se rechazan cuatro de los seis contrastes realizados de igualdad de parámetros. Esta moderación se encuentra entre los estudiantes de primer año (EXP_1) respecto a los de EXP_3 y EXP_4, los de segundo año (EXP_2) y los de EXP_3 y EXP_4. En el resto de las comparaciones no se rechaza la hipótesis nula de igualdad del parámetro estructural (C_S $\rightarrow$ C_G.C) con p-valor $>0.10$. Por lo que, entre los estudiantes de EXP_1-EXP_2 y EXP_3-EXP_4 no existen diferencias significativas.

Para expresarlo más concretamente podríamos decir que:

1) No existen diferencias significativas entre el primer y segundo año en la universidad $\left(E X P \_1:\right.$ C_S $\rightarrow$ C_G.C $=.0,26$ y EXP_2: C_S $\rightarrow$ C_G.C $=0,29 ; \chi 2[1]=0,67$ p $\left.>0,40\right)$.

2) Aunque el parámetro aumenta $(0,13)$, no existen diferencias significativas entre el tercer y cuarto año en la universidad (EXP_3: C_S $\rightarrow$ C_G.C $=0,40$ y EXP_4: C_S $\rightarrow$ C_G.C=0,53; $\chi 2[1]=2,29 p>0,13)$.

3) Sí existen diferencias significativas entre los estudiantes que están en primer curso y los que están en tercero y cuarto, respectivamente (EXP_1: C_S $\rightarrow C_{-}$G.C $=0,26$ respecto a EXP_3: C_S $\rightarrow$ C_G.C $=0,40, \chi 2[1]=5,33 p<0,05$, y EXP_4: C_S $\rightarrow$ C_G.C=0,53, $\chi 2[1]=13,67 p<0,00)$. 
4) Sí existen diferencias significativas entre los estudiantes que están en segundo curso y los que están en tercero y cuarto (EXP_2: C_S $\rightarrow$ C_G.C $=0,29$ respecto a EXP_3: C_S $\rightarrow$ C_G.C $=0,40, \chi 2[1]=2,99 p<0,10, y$ EXP_4: C_S $\rightarrow$ C_G.C $=0,53, \chi 2[1]=10,15 p<.00)$.

Por lo antes expresado, este efecto directo queda moderado por la experiencia del estudiante, y la relación entre calidad de servicio y calidad global de la carrera aumenta conforme aumenta la experiencia del estudiante, entonces existe evidencia para el no rechazo parcial de la Hipótesis 3.1a.

En lo que respecta a la Hipótesis $3.1 \mathrm{~b}$ cabe destacar que aunque los parámetros estimados primero aumentan y luego disminuyen (EXP_1: C_D $\rightarrow$ C_G.C $=0,40$, EXP_2: C_D $\rightarrow$ C_G. $C=0,44$, EXP_3: C_D $\rightarrow$ C_G.C=0,40, EXP_4: C_D $\rightarrow$ C_G.C=0,30), únicamente se rechaza una igualdad de parámetros al 10\% (EXP_2: C_D $\rightarrow$ C_G.C $=.44$ respecto a EXP_4: C_D $\rightarrow$ C_G. $C=.30, \times 2[1]=3.05 p<.10)$. El efecto de la calidad docente en la calidad global de la carrera disminuye parcialmente conforme aumenta la experiencia del estudiante, por lo que existe cierta evidencia para no rechazar la Hipótesis 3.1b.

La Hipótesis 3.2c se rechaza en la muestra analizada, esto es, los efectos directos entre calidad global de la carrera e intención positiva de recomendar la universidad estimados no son significativamente distintos atendiendo a las cuatro etapas consideradas (EXP_1:

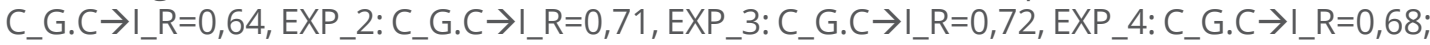
$\rightarrow \chi 2[1] p>0,44)$. Por lo tanto, este efecto directo no queda moderado por la experiencia del estudiante y la relación entre calidad global de la carrera e intención positiva de recomendar la universidad permanece estable a lo largo del tiempo en la muestra analizada.

Llegados a este punto nos planteamos valorar la jerarquía entre efectos, esto es, qué pesa más en la variable calidad global de la carrera: calidad de servicio facilitador o calidad docente. En la muestra total cabe no rechazar la hipótesis de igualdad de estos parámetros $\left(C \_S \rightarrow C \_G . C=C_{-} D \rightarrow C_{-} G . C=.37, \chi 2[1]=.00\right.$ p>.99). Por el contrario, sí cabe rechazar dicha hipótesis de igualdad al considerar únicamente aquellos buenos estudiantes que están en su segundo año en la Universidad (EXP_2: C_S $\rightarrow$ C_G.C $=0,29 \neq$ EXP_2: C_D $\rightarrow$ C_G.C $=0,44$, $\chi 2[1]=4,09 p<0,05)$, además de cuando están en su cuarto año en la Universidad (EXP_4: C_S $\rightarrow$ C_G.C $=0,53 \neq$ EXP_4: C_D $\rightarrow$ C_G.C $=0,30, \chi 2[1]=4,96 p<.0,05)$. Por ello existe evidencia de que no solo pueden cambiar dichos efectos a lo largo de los años sino que además la importancia puede invertirse. Al final del Primer Ciclo (segundo año en la Universidad) pesa más la percepción de la calidad docente $(0,29 / 0,44)$, mientras que al final del Segundo Ciclo (cuarto año) pesa más la calidad del servicio facilitador $(0,53 / 0,30)$.

Para finalizar, dados los resultados obtenidos se puede afirmar que los efectos indirectos de calidad de servicio y calidad de la docencia en intención de recomendar cambian a lo largo del tiempo (Tabla 5). Como en el caso anterior, al final del Primer Ciclo pesa más la calidad docente $(0,21 / 0,31)$, mientras que al final del Segundo Ciclo pesa más la calidad del servicio facilitador $(0,36 / 0,20)$.

\section{Conclusiones}

A partir de la revisión de la literatura sobre servicios y de educación superior se constató que las relaciones entre la calidad global, la calidad de la docencia y la calidad de los servicios facilitadores como antecedentes de la intención de recomendar la universidad no han sido estudiadas para los buenos estudiantes en educación superior. Este estudio propone un modelo de dichas relaciones con foco en los buenos estudiantes, con una visión multidimensional en su mirada multiservicios y dinámica en el tiempo. 
Respecto a los hallazgos, en primer lugar, en línea con las investigaciones de Athiyaman (1997), Paswan y Ganesha (2009) y Palmer et al. (2011), los resultados del presente trabajo aportan evidencia de que la percepción de la calidad global de la carrera es un antecedente positivo de la intención de recomendación de una universidad y, en línea con Rubinsztejn et al. (2015) la percepción de la calidad docente y de la calidad del servicio facilitador también presentan un efecto positivo en la percepción de la calidad global de la carrera. Por otro lado, los resultados ponen de manifiesto la importancia relativa del efecto de la calidad de los servicios facilitadores en la percepción de la calidad global de una carrera universitaria, resultados acordes con Teo y Soutar (2012) y con la teoría clásica de servicios de Grönroos (1990) que enfatiza la importancia de los procesos en la prestación de los servicios para asegurar la calidad global.

Más en profundidad, los buenos estudiantes dan idéntica importancia a los servicios facilitadores que a la docencia en la formación de la calidad global de la carrera universitaria, lo que se encuentra en línea con los resultados obtenidos por Petruzellis et al. (2006), García-Sanchis et al. (2015) y Wang (2012), que señalan la importancia de un análisis multidimensional del proceso. Esto es muy relevante dado el desarrollo creciente del aprendizaje a distancia en entornos virtuales y los sistemas de enseñanza MOOC, en los que se ha evidenciado que los servicios organizativos y administrativos caben ser considerados como determinantes en la percepción de la calidad global (Martínez-Argüelles et al., 2013). En concordancia con los resultados de Hill (1995), y atendiendo al dinamismo, al tenerse en cuenta la etapa de la carrera en la cual se encuentra el estudiante cambia el peso del efecto de las dimensiones servicio facilitador y docencia en la calidad de la carrera. Esto apoya la teoría de la evaluabilidad y la intangibilidad de los servicios que señala que los servicios facilitadores son más difíciles de evaluar en etapas iniciales de la carrera (Dagger y Sweeney, 2007; Rubinsztejn y Palacios, 2010).

Asimismo llama la atención una creciente importancia en el tiempo del peso de los servicios facilitadores, tanto en la percepción de la calidad global de la carrera como en la intención de recomendar la institución. Esto implica que las universidades no pueden descuidar los servicios facilitadores en etapas avanzadas del buen estudiante y deben identificar cómo hacerlo. Quienes toman las decisiones en las universidades deberían averiguar cómo debe ser el servicio facilitador para los buenos estudiantes en las etapas avanzadas de su carrera. Sin embargo, la magnitud del efecto de la percepción de la calidad de la carrera en la intención de recomendar se mantiene constante en las distintas etapas del programa educativo. En otras palabras, los buenos estudiantes no se vuelven más proclives a recomendar la universidad en las etapas finales de la carrera ante niveles similares de calidad de la misma. Esto podría deberse a que las expectativas del buen estudiante van aumentando cuando vamos cumpliendo con las mismas a medida que transcurre el consumo del servicio.

Concluimos entonces que aquellos que toman las decisiones en las universidades y que comprendan que las prioridades de percepción de calidad e intención a recomendar evolucionan en el tiempo, pueden tener una ventaja competitiva al diseñar una experiencia educativa y de servicio diferenciada para cada etapa. Estos hallazgos señalan la necesidad de las instituciones de educación superior de incorporar en su planificación estratégica la segmentación de los estudiantes según su experiencia. Asimismo, los que identifiquen y comprendan qué servicios facilitadores necesitan y priorizan a los buenos estudiantes en etapas avanzadas pueden beneficiarse de su fidelización en términos de predisposición positiva a recomendar la institución. A su vez pueden competir redefiniendo a los buenos estudiantes incluyendo aspectos funcionales de coproducción tal cual sugiere la escuela nórdica. 


\section{Limitaciones y futuras líneas de investigación}

En primer lugar cabe señalar que este trabajo no está exento de limitaciones. En nuestra opinión la principal tiene que ver con el instrumento de medida de los conceptos objetivo. El hecho de no haber empleado un cuestionario ad hoc ha limitado las posibilidades de utilización de un mayor número de ítems para medir las diferentes dimensiones de calidad del modelo. No obstante, esta limitación se ha intentado compensar mediante la aproximación metodológica empleada. La metodología utilizada en el contraste de las hipótesis, tal y como se ha descrito, son los Modelos de Ecuaciones Estructurales con un planteamiento multi-muestra, por lo que se ha considerado explícitamente el efecto moderador de la variable experiencia.

Una de las principales contribuciones de este estudio se concreta en la evidencia mostrada del carácter dinámico de los factores que influyen en la percepción de la calidad de la carrera y en la intención de recomendar a la universidad. Por lo que, desde nuestro punto de vista, resulta clave avanzar en nuevas investigaciones de carácter longitudinal que indiquen las variables concretas que conforman las dimensiones clave de percepción de los servicios facilitadores, profundizando en las expectativas y en los componentes clave de las diferentes dimensiones de la calidad. Si bien se ha establecido que los buenos estudiantes priorizan la calidad docente en etapas iniciales del proceso educativo y la calidad de servicio facilitador en etapas avanzadas de su carrera, aún se desconoce qué aspectos concretos de la docencia (materiales, tipo de casos, metodología didáctica, contenidos, feedback) y del servicio facilitador consideran relevantes estos buenos estudiantes.

Para finalizar se sugiere validar el modelo en universidades con características similares, así como en universidades con modelos de gestión diferentes o que estén actualmente transitando hacia el nuevo paradigma educativo centrado en el estudiante y en la educación a distancia. Principalmente porque, en coincidencia con las tendencias actuales respecto al aumento de los créditos electivos, la universidad sobre la que se realiza el análisis se caracteriza por contar con un alto número de asignaturas optativas y una metodología de enseñanza centrada en el aprendizaje que implica un alto nivel de involucramiento del estudiante en el proceso educativo. Esta particularidad es clave en lo que respecta a la generalización de los resultados ya que se trata de un caso en el que el estudiante es un coproductor intenso de la docencia y del servicio facilitador, condicionando la calidad de los resultados educativos (Kelley et al., 1990).

\section{Referencias bibliográficas}

Achterberg, C. (2005). What is an Honors Student? Journal of the National Collegiate Honors Council, 75-84.

Asparouhov, T. (2005). Sampling weights in latent variable modeling. Structural Equation Modeling, 12(3), 411-434.

Athiyaman, A. (1997). Linking student satisfaction and service quality perceptions: the case of university education. European Journal of Marketing, 31(7), 528-540.

Bentler, P.M. (2008). EQ86 Structural Equations Program Manual, Multivariate. Encino, CA: Multivariate Software Inc.

Bollen, K.A. (1998). Structural Equations Models. Wiley Online Library.

Bollen, K.A. (1989). Structural Equations with Latent Variables. New York: Wiley. 
Boyi, Q. (2006). Expectation, Service Quality, and Satisfaction in Higher Education (Tesis de maestría). Bedford: Cranfield University.

Bradshaw, G., Espinoza, S. \& Hausman, C. (2001). The College-Decision Making of High Achieving Students. College \& University Journal, 77(2), 15-22.

Brady, M.K. \& Cronin Jr., J.J. (2001). Some new thoughts on conceptualizing perceived service quality: a hierarchical approach. Journal of Marketing, 65(3), 34-49.

Brooks, R. (2006). Factors that influence traditional-age, high-achieving students to enroll at a research-extensive university in the Southern region of the United States (Tesis de doctorado). Baton Rouge: Louisiana State University.

Browne, M.W., Cudeck, R., Bollen, K.A. \& Long, J.S. (1993). Alternative ways of assessing model fit. Sage focus editions.

Bruce, G. \& Edgington, R. (2008). Factors influencing word-of-mouth recommendations by MBA students: an examination of school quality, educational outcomes, and value of the MBA. Journal of Marketing for Higher Education, 18(1), 79-101.

Brusoni, M., Damian, R., Sauri, J.G., Jackson, S., Kömürcügil, H., Malmedy, M. et al. (2014). The Concept of Excellence in Higher Education. European Association for Quality Assurance in Higher Education. Occasional Paper, 20, 1-44.

Chang, H.H., Jeng, D.J. \& Hamid, M.R. (2012). Conceptualising consumer's word-of-mouth behaviour intention: evidence from a university education services in Malaysia. Service Business, 7(1), 17-35.

Chapman, R.G. \& Jackson, R. (1987). College choices of academically able students: The influence of no-need financial aid and other factors. New York, NY: College Board Publications.

Chilcutt, A.M. (2014). Persuasion in Higher Education: Recruitment of High Ability Students (Tesis de grado). Hattiesburg: University of Southern Mississippi.

Clewes, D. (2003). A student-centred Conceptual Model of Service Quality in Higher Education. Quality in Higher Education, 9(1), 69-85.

Cook, R.W. \& Zallocco, R.L. (1983). Predicting university preference and attendance: Applied marketing in higher education administration. Research in Higher Education, 19(2), 197-211.

Dagger, T. y Sweeney, J. (2007). Service quality attribute weights: how do novice and longer-term customers construct service quality perceptions? Journal of Service Research, $10(1), 22-42$.

Dijk, G.H. (2015). Excellence in Higher Education (Tesis de grado). The Netherlands: University of Twente.

Elsharnouby, T.H. (2016). Participation behaviour among international students. The role of satisfaction with service augmentation and brand choice attainment. International Journal of Educational Management, 30(5), 679-697. 
García-Sanchis, M., Gil-Saura, I. y Berenguer-Contrí, G. (2015). Dimensionalidad del servicio universitario: una aproximación desde un enfoque de marketing. Revista iberoamericana de educación superior, 15, 26-49.

Gater, D.S. (2001). The Competition for Top Undergraduates by America's Colleges and Universities. Gainesville, Florida: The Center Reports.

Gibbs, G. (2010). Dimensions of Quality. York: The Higher Education Academy.

Grönroos, C. (1990). Service management and marketing: Managing moments of truth in service competition. Lexington, MA: Lexington books.

Grönroos, C. (2001). The perceived service quality concept - A mistake? Managing Service Quality, 11(3), 150-152.

Hair, J.F., Black, W.C., Babin, B.J., Anderson, R.E. \& Tatham, R.L. (2006). Multivariate Data Analysis ( $6^{\text {th }}$ ed.). Upper Saddle River, NJ.: Prentice-Hall.

Halai, N. (2013). Quality of private universities in Pakistan: An analysis of higher education commission rankings 2012. International Journal of Educational Management, 27(7), 775-786.

Helgesen, O. \& Nesset, E. (2007). What accounts for students' loyalty? Some field study evidence. International Journal of Educational Management, 21(2), 126-143.

Hill, M. (1995). Managing service quality in higher education: the role of the student as primary consumer. Quality Assurance in Education, 3(3), 10-21.

Hooper, D., Coughlan, J. \& Mullen, M. (2008). Structural Equation Modelling: Guidelines for Determining Model Fit. Electronic Journal of Business Research Methods, 6(1), 53-60.

Horstschräer, J. (2011). University Rankings in Action? The Importance of Rankings and an Excellence Competition for University Choice of High-Ability Students. Mannheim, Germany: Centre for European Economic Research.

Hu, L.T. \& Bentler, P.M. (1999). Cutoff criteria for fit indexes in covariance structure analysis: Conventional criteria versus new alternatives. Structural Equation Modeling: A Multidisciplinary Journal, 6(1), 1-55.

Jain, R., Sinha, G. \& Sahney, S. (2011). Conceptualizing service quality in higher education. Asian Journal on Quality, 12(3), 296-314.

Jöreskog, K.G. (1993). Testing structural equation models. In K. A. Bollen \&J. S. Long, Testing Structural Equation Models (pp. 294-316). Newbury Park, CA: Sage.

Kelley, S.W. (1990). Customer Participation in Service Production and Delivery. Journal of Retailing, 66(3), 315-336.

Kotler, P. \& Fox, K. (1995). Strategic Marketing for Educational Institutions. Upper Saddle River, NJ: Prentice-Hall. 
Litten, L.H. (1982). Different strokes in the applicant pool: Some refinements in a model of student college choice. The Journal of Higher Education, 53(4), 383-402.

Marsh, H.W. \& Roche, L. (1993). The use of students' evaluations and an individually structured intervention to enhance university teaching effectiveness. American educational research journal, 30(1), 217-251.

Martínez-Argüelles, M.J., Blanco, M. \& Castán, J.M. (2013). Las dimensiones de la calidad del servicio percibida en entornos virtuales de formación superior. RUSC Universities and Knowledge Society Journal, 10 (1), 89-106.

Marzo, M., Pedraja, M. \& Rivera-Torres, P. (2005). A new management element for universities: satisfaction with the offered courses. The International Journal of Educational Management, 19(6), 505-526.

Mazzarol, T. \& Soutar, G. (2002). "Push-pull" factors influencing international student destination choice. International Journal of Educational Management, 16(2), 82-90.

Mazzarol, T., Sweeney, J. C. \& Soutar, G. N. (2007). Conceptualizing word-of-mouth activity, triggers and conditions: an exploratory study. European Journal of Marketing, 41(12), 14751494.

McDougall, G.H. \& Levesque, T.J. (1994). A revised view of service quality dimensions: An empirical investigation. Journal of Professional Services Marketing, 11, 189-210.

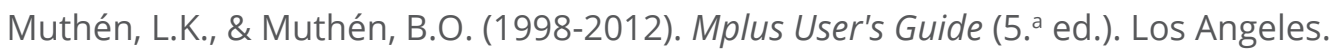

O'Neill, M.A. \& Palmer, A. (2004). Importance-performance analysis: a useful tool for directing continuous quality improvement in higher education. Quality assurance in education, 12(1), 39-52.

Palmer, J., Eidson, V., Haliemun, C. \& Wiewel, P. (2011). Predictors of Positive and Negative Word of Mouth of University Students: Strategic Implications for Institutions of Higher Education. International Journal of Business and Social Science, 2(7), 59-61.

Palmer, S.R. \& Holt, D.M. (2009). Examining student satisfaction with wholly online learning. Journal of computer assisted learning, 25(2), 111-113.

Paswan, A. K. \& Ganesha, G. (2009). Higher education institutions: satisfaction and loyalty among international students. Journal of Marketing for Higher Education, 19(1), 65-84.

Petruzzellis, L, D’Uggento A.M. y Romanazzi, S. (2006). Student satisfaction and quality of service in Italian universities. Managing Service Quality, 16(4), 349-364.

Quinn, A., Lemay, G., Larsen, P. \& Johnson, D.M. (2009). Service quality in higher education. Total Quality Management, 20(2), 139-152.

Ramsden, P. (1991). A performance indicator of teaching quality in higher education: The Course Experience Questionnaire. Studies in Higher Education, 16(2), 129-150.

Reiccheld, F.F. (2003). The one number you need to grow. Harvard Business Review, 81(12), 46-55. 
Renzulli, J.S. (2012). Reexamining the Role of Gifted Education and Talent Development for the $21^{\text {st }}$ Century: A Four-Part Theoretical Approach. Gifted Child Quarterly, 56(3), 150-159.

Rubinsztejn, G. \& Palacios, M. (2010). El efecto del tiempo en la percepción de la calidad del servicio educativo. Revista Iberoamericana de Educación, 54, 189-202.

Rubinsztejn, G., Rivera-Torres, P. \& Grijalvo, M.M. (2015). Calidad y recomendación en educación superior: el rol de la experiencia del estudiante. Interciencia: Revista de ciencia y tecnología de América, 40(12), 816-826.

Satorra, A. (1992). Asymptotic robust inferences in the analysis of mean and covariance structures. Sociologial Methodology, 22(1), 249-278.

Schoenherr, H.J. (2009). Beyond academic reputation: Factors that influence the college of first choice for high achieving students (Tesis doctoral). Florida: University of South Florida.

Spies, R. (1978). The Effect of Rising College Costs on College Choice: A Study of the Application Decisions of High-Ability Students. Research Report. Princeton. College Entrance Examination Board.

Stern, B.B. (1994). A revised communication model for advertising: Multiple dimensions of the source, the message, and the recipient. Journal of Advertising, 23(2), 5-15.

Sultan, P. \& Wong, H. (2010). Performance-based service quality model: an empirical study on Japanese universities. Quality Assurance in Education, 18(2), 126-143.

Swanson, S.R. \& Kelley, S.W. (2001). Service recovery attributions and word-of-mouth intentions. European Journal of Marketing , 35, 194-211.

Teo, R. \& Soutar, G.N. (2012). Word of mouth antecedents in an educational context: a Singaporean study. International Journal of Educational Management, 26(7), 678-695.

Vianden, J. \& Yakaboski, T. (2017). Critical incidents of student satisfaction at German universities. International Journal of Educational Management, 31(7), 944-957.

Wang, J. (2012). University Student Satisfaction in Shijiazhuang, China: An Empirical Analysis (Tesis de Maestría). New Zealand: Lincoln University.

Weiler, W.C. (1996). Factors influencing the matriculation choices of high ability students. Economics of Education Review, 15(1), 23-36.

White, C.J. (2011). On the evaluation of teaching and learning in higher education: a multicultural inquiry. Assessment \& Evaluation in Higher Education, 36(6), 643-656. 


\section{Notas}

* Universidad ORT Uruguay.

** Universidad Politécnica de Madrid (España).

*** Universidad de Zaragoza (España).

**** Universidad de la República (Uruguay).

${ }^{1}$ Doctor en Administración, Universidad Politécnica de Madrid (España). MBA, Esade, Barcelona (España). Ingeniero Civil, Technion Israel Institute of Technology. Certificado en Docencia Universitaria, Universidad ORT Uruguay. Consultor en Planificación estratégica de mercado, Goldfarb. Ex gerente de Comunicación e Imagen, Terra Networks Uruguay. Premio a la Excelencia Docente 2005 y Premio a la Trayectoria y Contribución 2016, Facultad de Administración y Ciencias Sociales, Universidad ORT Uruguay. Coordinador Académico de Marketing, Facultad de Administración y Ciencias Sociales, Universidad ORT Uruguay.

${ }^{2}$ Doctor en Ingeniería Mecánica y de Organización Industrial, Universidad Carlos III de Madrid (España). MBA y Ingeniero Industrial, Universidad Politécnica de Madrid (España).Profesora, Departamento de Ingeniería de organización, Administración de empresas y Estadística, Universidad Politécnica de Madrid (España).

${ }^{3}$ Doctora en Ciencias Económicas y Empresariales, Universidad de Zaragoza (España). Profesora, departamento de Dirección de marketing e Investigación de mercados, Facultad de Economía y Empresa, Universidad de Zaragoza (España).

${ }^{4}$ MBA,Universidad de la República (Uruguay). Máster en Marketing Digital y Comercio Electrónicom, EAE Business School (España). Profesor de marketing, Facultad de Ciencias Económicas, Universidad de la República (Uruguay).

\section{Contribución de autoría}

Los autores han realizado el artículo por partes iguales. 